\title{
A REVIEW ON A NOVEL APPROACH FOR DATA COLLECTION IN WSN
}

\author{
Ramandeep Kaur (1), Dinesh Kumar (2) \\ (1) Research Scholar, Department of Computer Science \& Engineering, GZSCCET, Bathinda \\ ramanhanda16@gmail.com \\ (2) Associate Professor, Department of Computer Science \& Engineering, GZSCCET, Bathinda \\ dk_scorpio@rediffmail.com
}

\begin{abstract}
Wireless sensor networks have become increasingly popular due to their wide range of application. Clustering sensor nodes organizing them hierarchically have proven to be an effective method to provide better data aggregation and scalability for the sensor network while conserving limited energy. Minimizing the energy consumption of a wireless sensor network application is crucial for effective realization of the intended application in terms of cost, lifetime, and functionality. However, the minimizing task is hardly possible as no overall energy cost function is available for optimization. In this paper, we have proposed a modified alogirthm of leach where hard and soft threshold values will be applied for improving the overall throughput and network lifetime.
\end{abstract}

\section{Keywords}

Wireless0 Sensor Networks, Protocol, Cluster Head, Base Station, Mobile node.

\section{INTRODUCTION}

A Wireless Sensor Network or WSN is supposed to be made up of a large number of sensors and at least one base station. The sensors are autonomous small devices with several constraints like the battery power, computation capacity, communication range and memory. They also are supplied with transceivers to gather information from its environment and pass it on up to a certain base station, where the measured parameters can be stored and available for the end user. In most cases, the sensors forming these networks are deployed randomly and left unattended to and are expected to perform their mission properly and efficiently. As a result of this random deployment, the WSN has usually varying degrees of node density along its area. Sensor networks are also energy constrained since the individual sensors, which the network is formed with, are extremely energy-constrained as well. The communication devices on these sensors are small and have limited power and range. Both the probably difference of node density among some regions of the network and the energy constraint of the sensor nodes cause nodes slowly die making the network less dense. Also, it is quite common to deploy WSNs in harsh environment, what makes many sensors inoperable or faulty. For that reason, these networks need to be fault-tolerant so that the need for maintenance is minimized. Typically, the network topology is continuously and dynamically changing, and it is actually not a desired solution to replenish it by infusing new sensors instead the depleted ones. A real and appropriate solution for this problem is to implement routing protocols that perform efficiently and utilizing the less amount of energy as possible for the communication among nodes. Due to recent technological advances, the manufacturing of small and low cost sensors became technically and economically feasible. The sensing electronics measure ambient conditions related to the environment surrounding the sensor and transforms them into an electric signal. Processing such a signal reveals some properties about objects located and/or events happening in the vicinity of the sensor. A large number of these disposable sensors can be networked in many applications that require unattended operations. A Wireless Sensor Network (WSN) contain hundreds or thousands of these sensor nodes. These sensors have the ability to communicate either among each other or directly to an external base-station (BS). Wireless Sensor Network (WSN) is a type of wirelessAd-Hoc network in which large numbers of sensor nodesare deployed in the application field [1]. Sensor nodes are inexpensive and low power devices.

Each node consists of four main units: Sensing Unit, ProcessingUnit, Communicating Unit and Power Unit. In sensingunit one or more sensors are placed to sense differentenvironment parameters like sound, temperature,vibration, pressure, motion and etc., based on applicationrequirement. Sensed data's (analog or digital) areprocessed and aggregated in the processing unit. The need for energy-efficient infrastructures for sensor networks is becoming increasingly important. Wireless sensor networks are networks consisting of many sensor nodes that communicate over a wireless media. A sensor node is equipped with a sensor module, a processor, a radio module and a battery. Since the battery limits the lifetime of the sensor nodes it also limits the lifetime of the sensor network, thus energy efficiency is a major issue for sensor networks. An important goal in many sensor networks is to monitor an area as long time as possible. Hence, it is important to distribute energy consumption evenly across the network. When the energy consumption is evenly distributed, the major part of the sensor nodes will stay alive approximately equally long time. This enables continued information gathering throughout the whole network area during the lifetime of the network. The most power-consuming activity of a sensor node is typically radio communication [2].]. Some sensor nodes become cluster heads and collect all traffic from their respective cluster. The cluster head aggregates the collected data and then sends it to its base station. When using clustering, the workload on the cluster head is thus larger than for non-cluster heads. The cluster heads should therefore be changed several times during the lifetime of the sensor network in order to distribute the extra workload and energy consumption evenly. 


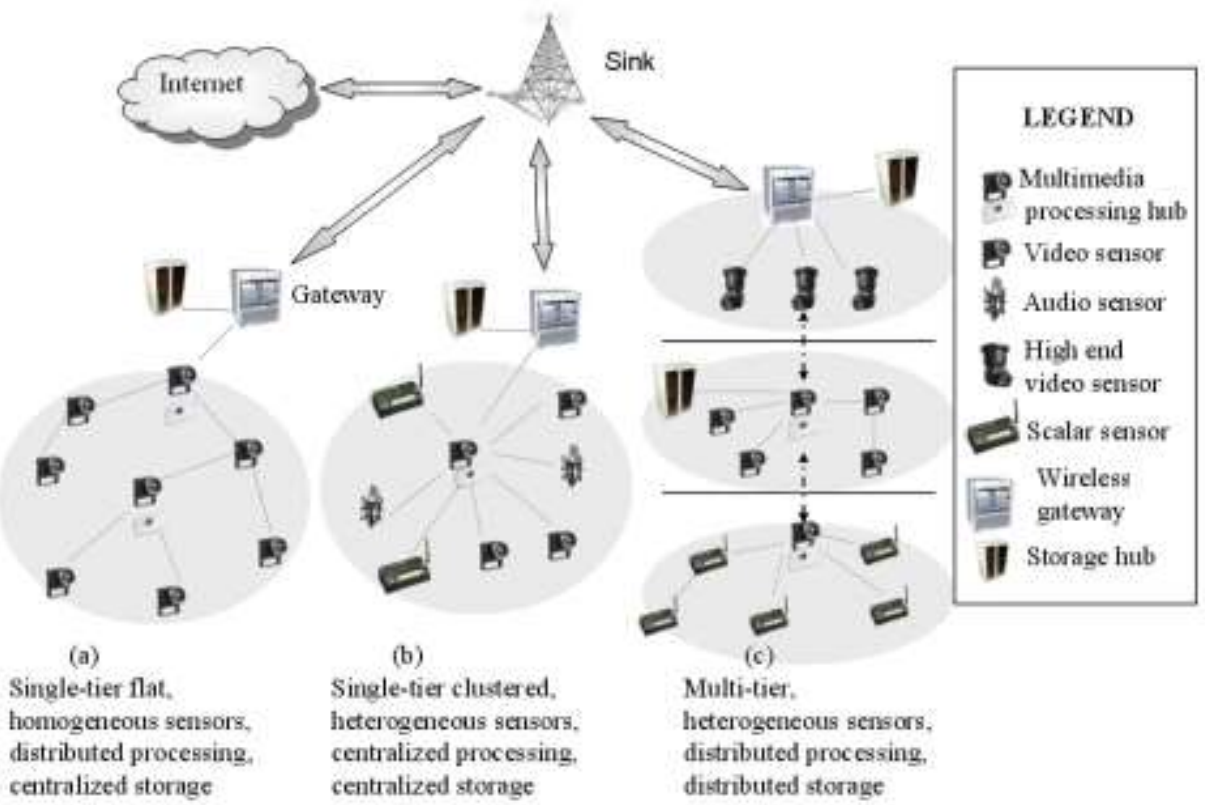

Figure 1. Wireless Sensor Network

Wireless sensor network is one of the category belongs to ad-hoc networks. Sensor network are also composed of nodes. Here actually the node has a specific name that is "Sensor" because these nodes are equipped with smart sensors [2]. A sensor node is a device that converts a sensed characteristic like temperature, vibrations, pressure into a form recognize by the users. Wireless sensor networks nodes are less mobile than ad-hoc networks. So mobility in case of ad-hoc is more. In wireless sensor network data are requested depending upon certain physical quantity. So wireless sensor network is data centric. A sensor consists of a transducer, an embedded processor, small memory unit and a wireless transceiver and all these devices run on the power supplied by an attached battery [1]

The WSN consist of two main components:

1. Sensor Nodes, and

2. Base Station (Central Gateway).

\section{SENSOR NODES}

Sensors nodes are typically built of few sensors and a mote unit as shown in Fig.1.2.A Sensor is a device which senses the information and pass it on to mote. Sensors are typically used to measure the changes in physical environmental parameters like temperature, pressure, humidity, sound, vibration and changes in the health parameter of person e.g. blood pressure and heartbeat. MEMS based sensor have found good use in sensor nodes. A mote consists of processor, memory, battery, A/D converter for connecting to a sensor and a radio transceiver for forming an ad hoc network. A mote and sensor together form a Sensor Node. A sensor network is a wireless ad-hoc network of sensor nodes. Each sensor node can support a multi-hop routing algorithm and function as forwarder for relaying data packets to a base station.

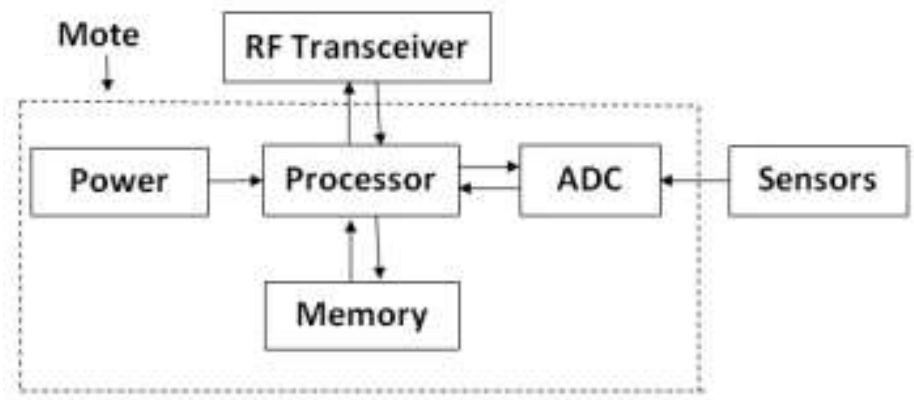

Figure 2. Block Diagram of Sensor Node

\section{BASE STATION}

A base station links the sensor network to another network. It consists of a processor, radio board, antenna and USB interface board. It is preprogrammed with low-power mesh networking software for communication with wireless sensor nodes. Deployment of the base station in a wireless sensor network is very important as all the sensor nodes handover their data to the base station for processing and decision making. Energy conservation, coverage of sensor nodes and 
reliability issues are taken care of during deployment of base station in sensor network. Generally base stations are assumed static in nature but in some scenarios they are assumed to be mobile to collect the data from sensor nodes.

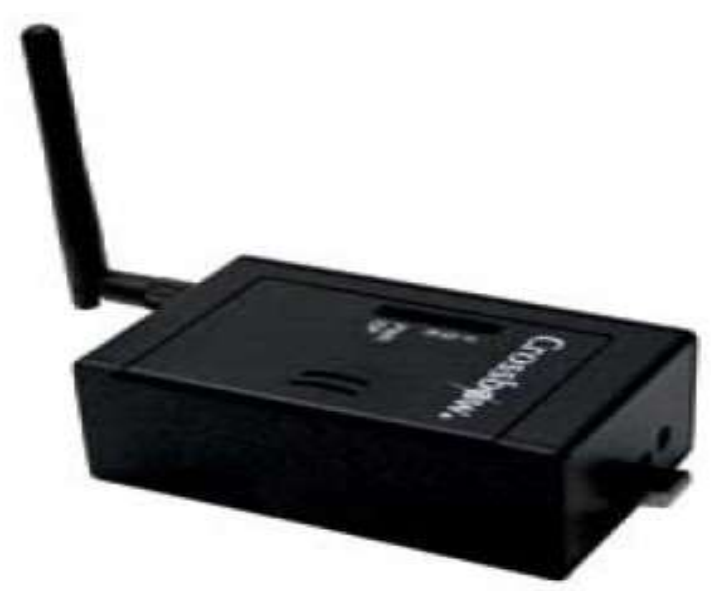

Figure 3. Base Station

\section{RADIO MODEL}

For the radio hardware, the transmitter dissipates energy to run the transmitter radio electronics and power amplifier, and the receiver dissipates energy to run the receive radio electronics as shown in Figure 4. For the scenarios described in this project work, both the free space (d2power loss) and the multi path fading (d4 power loss) channel models were used depending on the distance between the transmitter and the receiver. If the distance is less than a threshold, the free space (fs) model is used; otherwise, the multi path ( $\mathrm{mp}$ ) model is used.

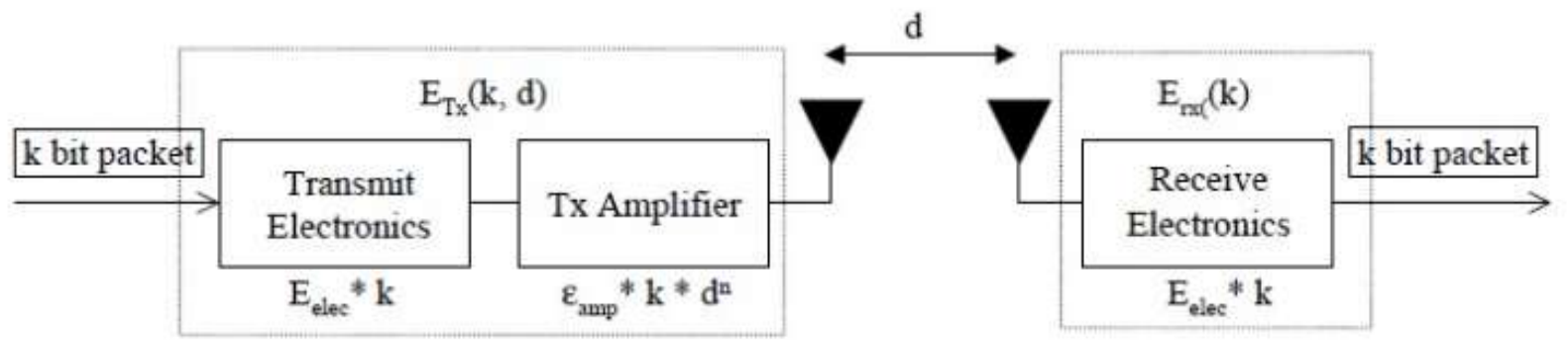

Figure 4. Radio Model

Wireless sensor networks have been evolved with the continuous advancements in embedded processing and wireless networking. Wireless sensor network is a class of wireless ad hoc networks in which sensor nodes collect, process and communicate data acquired from the physical environment to an external base station (BS), thus allowing for monitoring and control of various physical parameters [1]. Basically, wireless sensor network consists of a number of sensor nodes which work together to detect a region to capture data about the environment. The sensors are capable of communicating either among each other or directly to an external base station (BS). Each sensor node comprises sensing, processing, transmission, mobilization, position finding system, and power units. Many different routing, power management and data dissemination protocols have been designed till date for wireless sensor networks (WSNs), compatible with both the architecture of wireless sensor network (WSN) and the applications intended to support. These protocols support the practical existence of WSNs and efficiently make them an integral part of our lives in the real world.

\section{SENSOR NETWORKS APPLICATIONS}

Sensor networks may consist of many different types of sensors such as seismic, low sampling rate magnetic, thermal, visual, infrared, acoustic and radar, which are able to monitor a wide variety of ambient conditions that include the following [23]:

- temperature,

- humidity,

- vehicular movement,

- lightning condition,

- pressure, 
- soil makeup,

- noise levels,

- the presence or absence of certain kinds of objects,

- mechanical stress levels on attached objects, and

- the current characteristics such as speed, direction, and size of an object.

Sensor nodes can be used for continuous sensing, event detection, event ID, location sensing, and local control of actuators. The concept of micro-sensing and wireless connection of these nodes promise many new application areas. We categorize the applications into military, environment, health, home and other commercial areas. It is possible to expand this classification with more categories such as space exploration, chemical processing and disaster relief.

\section{ROUTING CHALLENGES AND DESIGN ISSUES IN WSNS}

Despite the innumerable applications of WSNs, these networks have several restrictions, e.g., limited energy supply, limited computing power, and limited bandwidth of the wireless links connecting sensor nodes. One of the main design goals of WSNs is to carry out data communication while trying to prolong the lifetime of the network and prevent connectivity degradation by employing aggressive energy management techniques. The design of routing protocols in WSNs is in uenced by many challenging factors. These factors must be overcome before e \pm cient communication can be achieved in WSNs. In the following, we summarize some of the routing challenges and design issues that a®ect routing process in WSNs.

Node deployment: Node deployment in WSNs is application dependent and affects the performance of the routing protocol. The deployment can be either deterministic or randomized. In deterministic deployment, the sensors are manually placed and data is routed through pre-determined paths.

Energy consumption without losing accuracy: sensor nodes can use up their limited supply of energy performing computations and transmitting information in a wireless environment. As such, energyconserving forms of communication and computation are essential. Sensor node lifetime shows a strong dependence on the battery lifetime [1].

Data Reporting Model: Data sensing and reporting in WSNs is dependent on the application and the time criticality of the data reporting. Data reporting can be categorized as either time-driven (continuous), event-driven, query-driven, and hybrid [13].

Node/Link Heterogeneity: In many studies, all sensor nodes were assumed to be homogeneous, i.e., having equal capacity in terms of computation, communication, and power. However, depending on the application a sensor node can have different role or capability. The existence of heterogeneous set of sensors raises many technical issues related to data routing.

Fault Tolerance: Some sensor nodes may fail or be blocked due to lack of power, physical damage, or environmental interference. The failure of sensor nodes should not affect the overall task of the sensor network. If many nodes fail, MAC and routing protocols must accommodate formation of new links and routes to the data collection base stations.

Scalability: The number of sensor nodes deployed in the sensing area may be in the order of hundreds or thousands, or more. Any routing scheme must be able to work with this huge number of sensor nodes

Network Dynamics: Most of the network architectures assume that sensor nodes are stationary. However, mobility of both BS's or sensor nodes is sometimes necessary in many applications [19].

\section{RELATED WORK}

The needed detailed literature survey, to get preliminary knowledge and search scope of investigation, to implement clustering is explained in this section. This synopsis presents investigational studies in several energy efficient routing algorithms and its general purpose.

Yu Xiuwu et al (2016) proposes a routing protocol that is based on monitoring area partition clustering for energy-balanced (EBPC). They divided monitoring area into several virtual partitions, controlled the number of common nodes and clusters by data fusion rate, and forwarded cluster heads data to the next partitions with minimum path selection coefficient. Samaresh Bera et al (2016) proposes a software-defined wireless sensor network architecture (Soft-WSN)-an effort to support application-aware service provisioning in Internet of Things (IOT). Detailed architecture of the proposed system is presented involving the application, control, and infrastructure layers to enable software-defined networking (SDN) in loT.

Alduais et al (2016) specifies that WSN has become an enabler technology for the IOT applications which extend the physical reach of the monitoring capability. WSN as it is, possess several constraints such as limited energy availability, low memory size, and low processing speed, which are the principal obstacles to designing efficient management protocols for WSNs, what more if it concerns WSN-IOT integration. Omkar Singh et al (2016) compares three well accepted WSN routing protocols namely LEACH, FAIR and SEP for their energy pattern in heterogeneous scenario. Heterogeneity is introduced in terms of initial random energy values given to the sensor nodes. All simulations are done in MATLAB. Different parameters are used for checking the efficacy of the considered routing protocol for H-WSN. Rani Kumari et al (2016) analyzes the performance of the various routing protocols of the wireless network in the WBAN and WSN. Moreover, the paper also compares their performance in the same network and in different network using different parameters i.e. PDR, latency and throughput etc. The research signifies that the wireless protocols needs updating to 
perform well in the WBAN. Hector Kaschel et al (2016) describes the mechanisms to achieve energy efficiency in a WSN, focusing particularly on routing protocols. Based on the state of the art, is presented a complete taxonomy of routing protocols used in WSN exposing their level of energy efficiency. Asdianur et al (2016) studied effectiveness in using Compressive Sensing (CS) algorithm in order to reduce measuring in IEEE 802.15.4 Standard Wireless Sensor Network (WSN). As well known, in common WSN work system, Base Station (BS) gather some information from available nodes, which the process itself consumes a lot of energy from each node. Mohd Zaki Shahabuddin et al (2016) proposes fundamental modelling of topology control algorithm to conserve individual WSN node's energy, and at the same time preserving the graph connectivity. The proposed topology control algorithm consists of three phases: 1 . Identifying connecting nodes at maximum transmission, 2. pairing nodes with shortest algorithm/minimum energy level, 3. Calculating/setting minimum power transmission per-node for energy conservation. The algorithm works-out locally and dispenses full graph connectivity, and theoretically would be able to reduce WSN control overhead. Abhay kumar et al (2016) presents a novel scheme to improve the lifetime of a sensor node by optimizing the size of the packet such that there is no loss of data along with providing an increased lifetime for the nodes. Wireless sensor network is a rapid developing area with diverse applications. Smartness and interoperability of network keeps it in high demand and hence comes need for the efficiency of the system. The most important limitation on sensor node is the low power consumption. Sensor nodes carry inadequate, generally irreplaceable power sources. Therefore, while traditional networks aim to achieve high quality of service (QoS), wireless sensor network protocols must emphasis predominantly on power conservation.

B. Bengherbia et al (2016) states that the wireless sensor network (WSN) represent fertile area for research and exploration, especially with enormous development in modern electronic systems, where it becomes an alternative to traditional wired systems. The wireless stems are less energy-consumptive and inexpensive, in addition it helps to reduce the complexity of wiring. Wireless sensor networks require the design of high density computing, an energy-efficient and a flexible node architecture. Gagandeep Kaur et al (2016) decreases the passive attack on sink node by decreasing the traffic on sink node. The simulation results demonstrate the proposed method can each node will compress their data before sending to cluster head. After compressing, the packet size of node will decrease. This will decrease the traffic overload. Mei Wu et al (2016) proposes a new model to efficiently control cluster-heads using heterogeneous sensors in real-time. It also proposes a generic software framework to feasibly and efficiently meet the needs of the users and applications (e.g., reliability, energy efficiency). The proposed model and its method have been implemented and evaluated in MATLAB, comparing its performance with known protocols for WSNs. The proposed model is suitable for common use because it is superior to other methods by energy efficiency. Sanaa. $S$ et al (2016) introduces an authentication protocol and simple key distributed scheme between sensor nodes. Node mobility has been taken into consideration and the work proposes a re-authentication protocol that is very efficient than the initial protocol. A wireless sensor network (WSN) consists of thousands of sensors and one base station. Sensors are deployed in the network to monitor target area and sense information according to the applied application then send this information to the base station. Roman Lara-Cueva et al (2016) presents a study in order to identify the value range of the main parameters within Carrier Sense Multiple Access (CSMA) defined in IEEE 802.15.4 that guarantee a satisfactory Wireless Sensor Networks (WSN) performance for a possible volcano monitoring application. Moreover, this study performs the comparison between test-bed in outdoor scenarios with the purpose of distinguishing the optimal number of nodes or each gateway according the main constrains given by a existing sensor network for real-time (RT) volcano monitoring system such as sampling time, packet loss, and delay.

\section{RESEARCH GAP}

Due to the deployment of WSNs in unattended harsh environment conditions, it gets to be unrealistic to charge or replace their batteries. Accordingly, the energy efficient operation of wireless sensors is very important to prolong the lifetime of overall wireless sensor networks. Furthermore, in WSN, there is wireless communication among all the nodes. Moreover, the energy consumed to transmit a message is exceptionally more than the energy needed to receive the same message. Besides all this, the route of each message destined to the base station too decides the network lifetime; for example, any node with depleted battery in short routes to the base station may lead to decreased network lifetime. While, using a long route composed of many sensor nodes can significantly increase the network delay.

- To decide cluster area on the basis of chosen cluster head consumes huge amount of energy resources in the communication between nodes for the purpose of fixing the cluster region and its head. When two cluster heads are very close to each other that means their ranges will overlap each other which will create a major problem.

- $\quad$ No parameter has been explained for the election of Cluster head. There are several parameters that are involved in the election of cluster head.

- $\quad$ Same level of power is used to amplify the signals from member node to cluster head, cluster head to mobile node and mobile node to base station. This will consume lot of energy.

- Mobile node requires large amount of energy for transferring the data of complete sector to the base station.

\section{CONCLUSION}

Sensor nodes are the main component of sensor network. Their main objectives are making discrete, local measurement about phenomenon surrounding these sensors, forming a wireless network by communicating over a wireless medium, and collect date and rout data back to the user via sink In this paper, we have reviewed several mechanisms for minimizing the total energy consumption of wireless sensor network applications for improving the overall network lifetime. Various research gaps have been formulated and will be further resolved using Matlab environment. 


\section{REFERENCES}

[1] Y. Xiuwu, Fan Feisheng Zhou Lixing and Z. Feng, "WSN Monitoring Area Partition Clustering Routing Algorithm for Energy-Balanced," IEEE, pp. 80-84, 2016.

[2] S. Bera, S. Misra, Sanku Kumar Roy and Mohammad S. Obaidat, "Soft-WSN: Software-De?ned WSN Management System for loT Applications," IEEE, pp. 1-8, 2016.

[3] N. A. M. Alduais, J. Abdullah, J. Abdullah, A. Jamil and L. Audah, "An Efficient Data Collection and Dissemination for IOT based WSN," IEEE, 2016.

[4] O. Singh, V. Rishiwal and M. Yadav, "Energy Trends of Routing Protocols for H-WSN," IEEE, 2016.

[5] R. Kumari and. P. Nand, "Performance Comparison of various Routing Protocols in WSN and WBAN," IEEE, pp. 427-431, 2016.

[6] Hector Kaschel and ohanna Ortega , "Energy efficiency in routing protocols applied to WSN," IEEE, 2016.

[7] Asdianur Hadi and Ida Wahidah, "Delay Estimation using Compressive Sensing on WSN IEEE 802.15.4," IEEE, pp. 192-197, 2016.

[8] Mohd Zaki Shahabuddin, Halabi Hasbullah and Izzatdin A Aziz, "eliminary Framework of Topology Control Algorithm Ahieve Node's Energy Efficiency," IEEE, pp. 259-263, 2016.

[9] Abhaykumar L. Gupta and Narendra Shekokar, "A Novel Approach to Improve Network Lifetime in WSN by Energy Efficient Packet Optimization," IEEE, 2016.

[10] B. Bengherbia, S. Chadli, M. Ould Zmirli and A. Toubal, "A MicroBlaze Based WSN Sink Node Using XBee Transceiver," IEEE, pp. 831-834, 2016.

[11] Gagandeep Kaur, Deepali and Rekha Kalra, "Improvement and Analys Security of WSN From Passive Attack," IEEE, pp. 4520-425, 2016.

[12] M. Wu, H. Liu and Q. Min, "Lifetime Enhancement by Cluster Head Evolutionary Energy Efficient Routing Model for WSN," IEEE, pp. 545-548, 2016.

[13] Roman Lara-Cueva, Rodolfo Gordillo, Liliana Valencia and Diego S. Ben, "Determining the Main CSMA Parameters for Adequate Performance of WSN for Real-time Volcano Monitoring System Applications," IEEE, pp. 1-9, 2016.

[14] Sanaa. S. Abd El dayem and M. R. M. Rizk , "An Efficient Authentication Protocol and Key Establishment in Dynamic WSN," IEEE, pp. 178-182, 2016. 